\title{
Self-Repair of Cooperation Network under Nodes Failure
}

\author{
Yaofeng Zhang ${ }^{1,2, *}$ and Renbin Xiao ${ }^{2}$ \\ ${ }^{1}$ Institute of Systems Engineering, Huazhong University of Science and Technology, Wuhan 430074, China \\ ${ }^{2}$ Statistic and Applied Mathematics Department, Hubei University of Economics, Wuhan 430205, China
}

Received: 6 Jun. 2013, Revised: 9 Oct. 2013, Accepted: 10 Oct. 2013

Published online: 1 Jan. 2014

\begin{abstract}
To further study the influences of nodes failure on the total fitness degree and the cooperative ratio in cooperation network, we established the cooperation network model firstly, and then, the update rules of individual strategy and the rules of networks selfrepair are constructed. The process of self-repair in cooperation network under nodes failure is analyzed by the agent-based simulation experiments. The results show that the nodes failure not only reduces the total fitness degree, but also reduces the cooperative ratio in the cooperation network. However, by introducing the self-repair rules, the emergence of a few new cooperative nodes can soon make up the loss and improve the total fitness degree and cooperative ratio gradually. The further analysis show that, the number of new nodes which to make the fitness degree return to the previous level are less than $20 \%$ of the failure nodes.
\end{abstract}

Keywords: self-repair, nodes failure, cooperation network, Agent-based method

\section{Introduction}

The complex networks [1,2] can be divided into social networks, information networks, technology networks and biological networks [3]. Social networks where individuals are linked with each other by cooperation is called cooperation network [4] . The individual in cooperation network are generally a single person, and may also be a group of people, a corporation, a company, and so on. The most famous cooperation network includes actor cooperation network [5] , scientist cooperation network [6], and so on.

As a kind of complex system, social cooperation network is constantly evolving. The network structure can be regard as the evolvement of members' cooperative relationship. In cooperation network, the individuals can choose their strategies between cooperation and un-cooperation. After Axelord brought repeated PD game model to the research of cooperation, the evolutionary game theory has been widely applied as a significant tool to study social cooperation [7] . In cooperation network, PD game exists in many aspects such as information sharing and products pricing [8], so the repeated PD game model has become the standard model for studying the cooperation network. Nowadays, based on PD game model, many scholars have studied the emergence and dynamic evolution of cooperation on different network topology structures $[9,10,11]$.

In cooperation network, individuals may secede from the cooperation network for the outside interference. When the nodes failure causes the large node vacancy in cooperation network, the cooperation interrupted. The interference may come from economic crises, policy changes and other social factors, and may also come from terrorist attacks, natural disasters and other emergencies. The failure of nodes influences the structure of the cooperation network to a certain extent. Nowadays, the research of the influence of nodes failure on cooperation network focuses on microcosmic strategy of emergency management [12]. Some other scholars study strategic evolution in cooperation network considering the systematic science [13]. In addition, various mechanisms affecting cooperation are also explored from a wide perspective of complex network [14] . Although previous literatures have researched on cooperation of social networks from different sides, nodes failure which is an influential factor with wide realistic significance is seldom discussed. With the development of economic globalization, the cooperation network becomes more and more popular. So it is significant to treat with nodes failure caused by different emergencies.

In this paper, the update rule of individual strategy is constructed on the basis of the cooperation network

\footnotetext{
*Corresponding author e-mail: zyfgzl@ sina.com
} 
model, and the Agent-based model in cooperation network is established with nodes failures. By the simulation experiments, the influence of the self-repair process of cooperation network with nodes failure is simulated and analyzed.

\section{The model and algorithm}

\subsection{Social cooperation networks}

Cooperation network is a special social network in which individuals are linked through cooperation. For example, in scientific cooperation network, each researcher is regarded as a vertex. That two vertexes are linked by a line denotes two researchers have published an article together. In general, the entire structure of cooperation network has a characteristic of complex network.

Individuals in cooperation network may come from the different characteristic groups (Figure 1). For instance, scientists in scientific cooperation network may come from the same field, and may come from the different, and enterprises in virtual enterprise network may belong to different value chain. In this paper, we take cooperation network with two different kinds of characteristic nodes as an example. Individuals in network form a certain cooperative by a long-term interaction. Every individual has different number of partners and different abilities of gaining information. The network model is built in the following way:

1. Form an initial cooperation network with $n=n_{1}+n_{2}$ nodes, where $n_{1}, n_{2}$ denotes the nodes' number of group 1 and group 2 respectively.

2. Suppose the individuals in cooperation network have formed a fixed cooperative relationship. $p_{i j}(i=1,2$; $j=1, \ldots n_{i}$ ) denote the individuals' capacity of selecting partners, and they reflect individuals' reputation, strength and other indexes. $q_{i j}\left(i=1,2 ; j=1, \ldots n_{i}\right)$ denote the individuals' capacity of studying from neighbors, namely, the ability of gaining information.

3 . Individuals are randomly matched by $p_{i j}$, and then the cooperation network is formed.

\subsection{PD Game Model}

In the PD game, each of the two players either cooperate $(C)$ or defect $(D)$. We assume that a cooperator pays a cost $\mathrm{c}$ for another individual to receive a benefit $b(b>c)$, and a defector pays no cost and does not distribute any benefits. Thus the reward for mutual cooperation is $R=b-c$, the sucker's payoff $S=-c$, the punishment for mutual defection is $P=0$, and the temptation to defect is $T=b$. And then, the beneficial matrix can be written as:

$$
\left(\begin{array}{cc}
b-c, b-c & -c, b \\
b,-c & 0,0
\end{array}\right)
$$

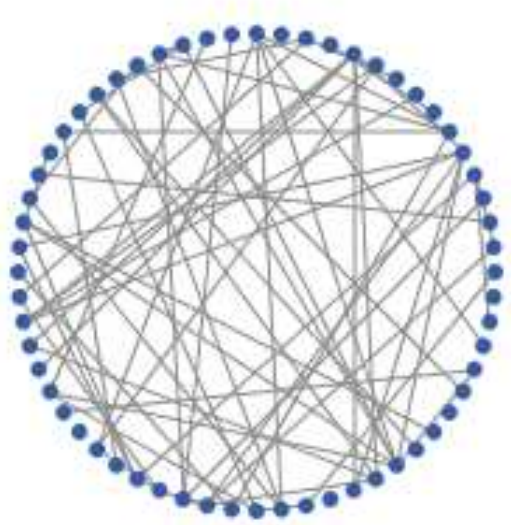

(a) Single-group

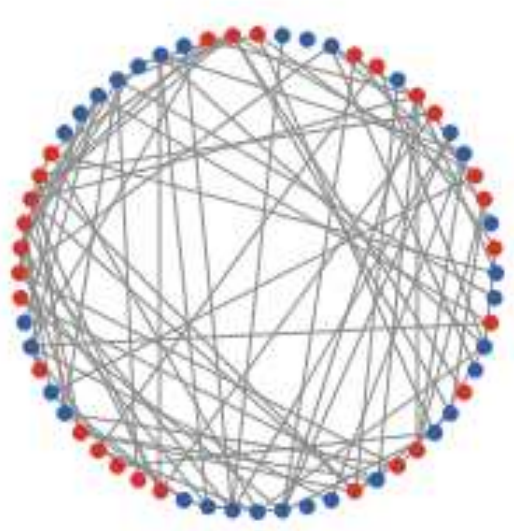

(b) Two-group

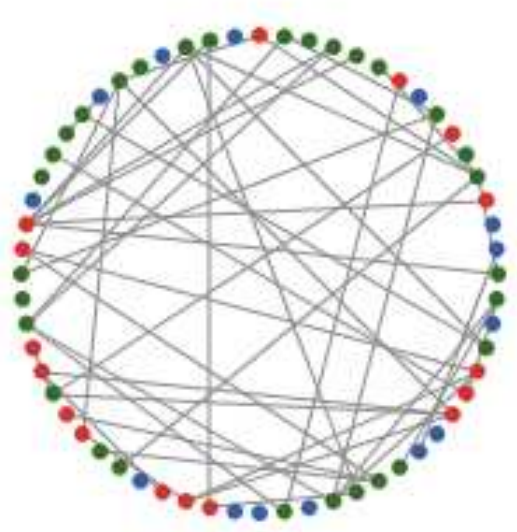

(c) Multi-group

Fig. 1: Different social cooperation networks 
Following reference [10], without loss of generality, the payoffs to the case of PD game are rescaled such that $R=1, T=1+r, S=-r$, and $P=0$, where $r=c /(b-$ $c)$ denotes cost-benefit rate. So the above model can be transformed into a single variable form just as:

$$
\left(\begin{array}{cc}
1,1 & -r, 1+r \\
1+r,-r & 0,0
\end{array}\right)
$$

Though Nash equilibrium strategy of the one-off PD game is $(D, D)$, according to Axelrod's theory [15] , cooperation can come forth in the PD game with unlimited or the game with unknown deadline.

\subsection{The Update of Individual Strategy}

Cooperation network is a complex system, its structure constituted by individuals' intercourse, so it's not static system. By game and study with partners, individual updates his game strategy continually, and then adjusts his relationship with others. This kind of strategy update among individuals promotes the emergence of the holistic cooperative characteristic in network.

Suppose that each node only games with the nodes of other group, and only learn strategy from the nodes in the same group. In one game, the sum of the incomes of each node is assumed as $U_{i}=\sum_{j \in \Omega_{i}} K_{i j}$, we call the $U_{i}$ as fitness function. Where $\Omega_{i}$ denotes the game neighbors of node $i$, and $K_{i j}$ is the incomes of $i$ game with node $j$. Every node chooses one learning neighbor to compare their fitness degrees. Because someone is always looking for higher benefit, so the strategy of node with high benefit is easily imitated by others. So we suppose nodes update their strategies by following rule [14] :

$$
\Pi_{i j}=\frac{1}{1+\exp \left[\frac{\left(U_{i}-U_{j}\right)}{k}\right]}
$$

Where $\Pi_{i j}$ denotes the probability of node $i$ taking the strategy of $j, U_{i}$ and $U_{j}$ denotes the incomes of the $i$ and $j$ respectively. And $k$ is noise coefficient, which denotes the strategy of node with low benefit may be imitated with little possibility because the bounded rationality.

Define the network's cooperative ratio as:

$$
\rho_{c}=\frac{n_{c}}{n}
$$

Where $n_{c}$ denotes the number of cooperative nodes.

We define the total fitness degree $U$ as the sum of all nodes' fitness degree.

$$
U=\sum_{i=1}^{n} U_{i}
$$

Define the best state of social cooperative network as:

$$
f=f\left(\rho_{c}, U\right)
$$

$f$ is decided by both the cooperative ratio and total fitness degree.

\subsection{Self-repair rules}

Let $L_{n}=\left[a_{n}, a_{n-1}, \cdots, a_{1}\right]$ denotes the set of the total fitness degree obtained within the latest $n$ evolvements, and $a_{i}$ be the total fitness degree obtained in the latest $i$ evolvements. When network evolves to stabilization, the mean value of $L_{n}$ denotes the steady fitness degree, marked as $Q$. Let system's steady criterion is

$$
\operatorname{Max}_{n}-\operatorname{MinL}_{n}<e
$$

Where $\operatorname{MaxL}_{n}$ and $\operatorname{Min} L_{n}$ denote the maximum and minimum value of $a_{i}$ in $L_{n}$ respectively, and $e$ is restrict constant.

Before nodes failure, nodes game and update strategy in the light of rule (2) and (3). Suppose the steady fitness degree is $Q_{1}$ before nodes failure. After nodes failure, nodes disappear randomly according to interference degree $M$.

$$
M=\frac{m_{1}+m_{2}}{n}
$$

Where $m_{1}, m_{2}$ denote the numbers of disappearance nodes of different kinds of group in cooperation network, and $n$ is the total number of network's nodes.

The cooperative network's primary structure is destroyed by nodes failure, and then the surviving nodes find the new game neighbors and learning neighbors. When system retunes to stabilization, we calculate the steady fitness degree once again, and note it as $Q_{2}$. Comparing $Q_{2}$ with $Q_{1}$, if $Q_{2}<Q_{1}$ we let the system spring out a new node, and then initialize its $p_{i j}$ and $q_{i j}$. In fact, there are many examples in real life, such as a new movie star, a new scholar or a new enterprise and so on. Generally speaking, the new node tends to choose cooperative strategy in first time, so as to receive its honor. So we suppose the new nodes' initial strategy is cooperation, and the new node chooses game neighbors and learning neighbors according to their $p_{i j}$ and $q_{i j}$. When new node chooses game neighbors, their reputation is a significant factor, so we let the new node chooses randomly several old nodes as game neighbors which take cooperative strategy within the near three games.

When system retunes to stabilization again, calculate $Q_{2}$ and compare with $Q_{1}$, if $Q_{2}$ still small then $Q_{1}$, a new node comes forth. This process is repeated until the system attains the original steady fitness degree.

\subsection{The Algorithm}

Using Agent-based method, we build an environment system for simulation experiment. During the course of the simulation, every individual plays with its interaction neighbors and gains the payoff firstly. And then, by comparing with the payoff of one of its learning neighbors and itself, the individual adopts the strategy of its neighbor with the probability $\prod_{i j}$. When the system 
tends to stable, we make some nodes disappear to reflect the nodes failure, and then hatch the new nodes according the self-repair rules until the cooperation network return to the original condition. The algorithm of model is list in table 1 .

Table 1: The general algorithm of model

ALGORITHM The general algorithm for the self-repair of
cooperation network under nodes failure, as given in the text.
(1) create initial nodes
(2) initial the nodes' parameters of $p_{i j}$ and $q_{i j}$
(3) create the cooperation network according to $p_{i j}$
(4) for each individual, calculate the payoff $U_{i}$ according to
matrix (2) and update strategy according to formula (3)
(5) when system tends to stable (according to formula (7)),
calculate $Q_{1}$, and make some nodes disappear randomly
(6) create one new node and initial it's $p_{i j}$ and $q_{i j}$
(7) when the system tends to stable again, calculate the $Q_{2}$
(8) compare $Q_{2}$ and $Q_{1}$, if $Q_{2}<Q_{1}$,repeat step (6) to (7),
otherwise, stop

\section{Simulation Experiment and Analysis}

In follow paragraph, the dynamic evolution of cooperation with nodes failure is discussed with the effect of network's self-repair rule. And then, the relationship between steady network fitness degree and interference degree with nodes failure, and the relationship between interference degree and the number of new nodes required by network selfrepair are given. The network's self-repair rule with nodes failure operates according to the definition in paragraph 2.4 .

In the simulation experiment below, the evolution of cooperation are analyzed with nodes failure. We take 1000 initial network nodes and assume that the number of individuals in every group is equal in all networks. Let $0 \leq p_{i j} \leq 12,0 \leq q_{i j} \leq 12, k=0.1, r=0.1, e=3000$, and take $n=100$ to record the results of $L_{n}$. The node's game benefits are calculated by game matrix (2). In initial states, each individual chooses his game neighbors and learning neighbors randomly by neighbors' $p_{i j}$ and $q_{i j}$. When the system reaches stable, $m$ nodes disappear randomly to simulate the emergence of nodes failure.

It can be seen from Figure 2 that network's total fitness degree becomes steady when system evolves to 200 steps. Now select 50\% nodes and make them disappear to simulate the nodes failure. From the following networks evolution, it's shown that, nodes failure reduces the network's total fitness degree rapidly.

By many simulation experiments, it is found that, in cooperation network, nodes failure can not only decrease the total cooperative fitness degree, but also reduce the network cooperative ratio slightly. Figure 3 shows that when interference degree is $50 \%$, nodes failure reduces

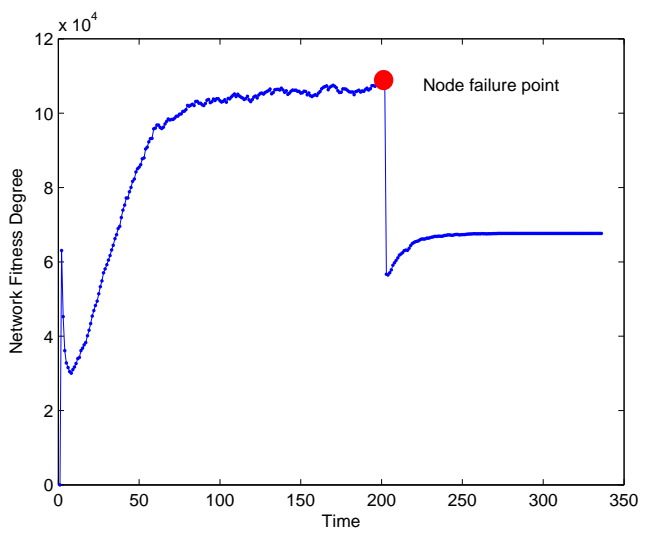

Fig. 2: Influence of nodes failure on the fitness degree

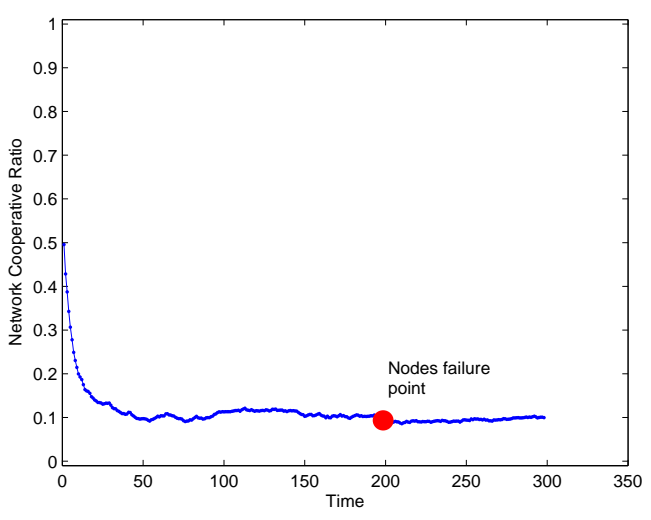

Fig. 3: Influence of nodes failure on the network's cooperative ratio

cooperative ratio slightly in cooperation network. The reason why the network's cooperative ratio depresses is that nodes failure weakens the heterogeneity of network. And the reduction of heterogeneity is not benefit for cooperation [16]. Hence, nodes failure causes the reduction of cooperative ratio in cooperation network.

In fact, nodes in real cooperation network are not static. With the nodes failure, some original nodes disappear, many new nodes come forth subsequently, and then cooperation network returns to stable through nodes' growth and the priority connection rules.

In order to further study this situation, in follow paragraph, we take 2000 initial network nodes, $0 \leq p_{i j} \leq 12,0 \leq q_{i j} \leq 8, e=300$ and keep other parameters unchanged. When the initial cooperation network evolves to be stable, we let nodes failure with the interference degree $m=0.5$. From Figure 4, we can see that the total fitness degree decreases suddenly. Subsequently, the continuous participation of new nodes which choose cooperation as initial strategy increases the 


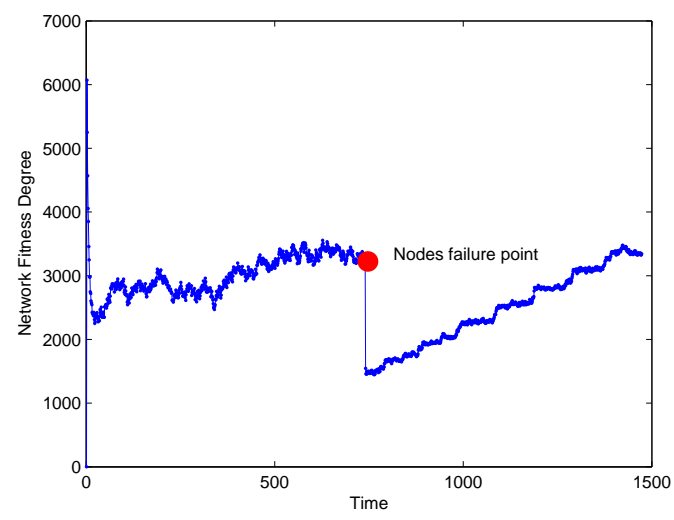

Fig. 4: Network total fitness degree changes with time after introducing the network's self-repair rule

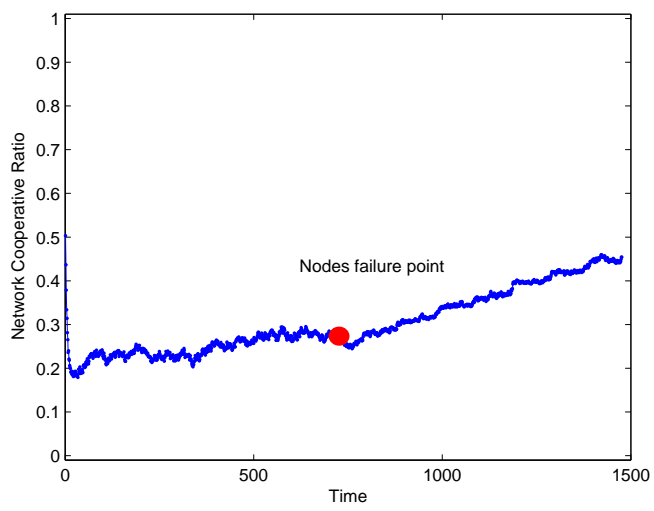

Fig. 5: Cooperative ratio changes with time after introducing network's self-repair rule

total fitness degree steadily till the original state before nodes failure. The cooperative ratio decreases after nodes failure, but then increases gradually (Figure 5), which is different from the situation without the self-repair rule. The reason why the total fitness degree and cooperative ratio can both increase steadily is that the new added nodes make the total number of nodes increase constantly, which increases the total fitness degree. On the other hand, the initial strategies of the new nodes are all cooperation and they always connected with the reputed existing nodes. When network reaches to be stable again after nodes failure, new nodes' participation help the terrible-state network.

Figure 6 shows the comparison with the number of new added nodes which required to help the network to regain the previous fitness degree and the number of disabled nodes which caused by nodes failure. We note that the more being destroyed of network, the more nodes are required to repair the total benefits back to the

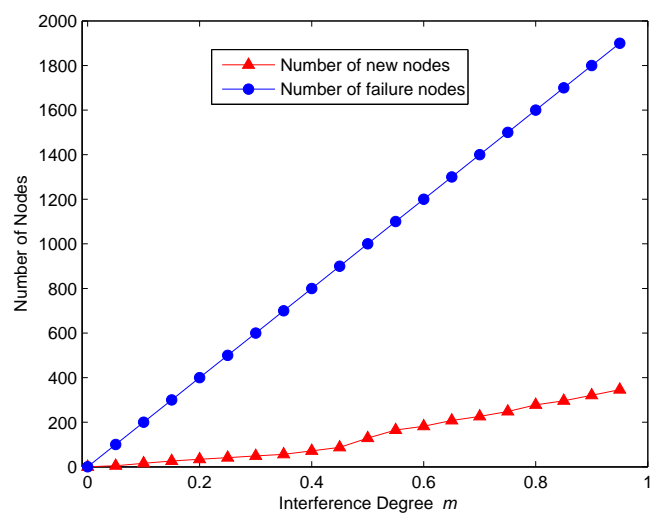

Fig. 6: Number of new added nodes required for network's self-repair change with interference degree

previous level before nodes failure. But the new added nodes are far less than the disabled nodes. The new added nodes don't exceed $20 \%$ of disabled nodes. What suggests further in Figure 6 is that, as the new added nodes always take part in initial game by cooperation and they always choose reputed nodes as their game and study neighbors, so when the destroyed network tends to stable state, the new cooperative nodes break the balance, which makes the network system tend to the cooperation. Hence, few new cooperative nodes will break stability in a relatively balanced state and tend to cooperation, which finally increases the total fitness degree and cooperative ratio.

\section{Conclusion}

Nowadays, financial crisis and terrorist attack break out frequently. So the cooperation network can be easily affected and cause nodes failure. In this paper, the dynamic evolution model in cooperation network with nodes failures is established on the basis of the cooperation network structure and the Agent-based method. As the structure of the cooperation network is actually the structure of individuals' cooperation, so the structure of the cooperation network is destroyed with nodes failure while the original cooperation is ruined. The results of the simulation experiment show that nodes failure decreases not only the total fitness degree but also cooperative ratio. However, when the self-repair rule is further introduced to the cooperation network, the total fitness degree and cooperative ratio can both increase rapidly. And it just needs new added nodes, less than $20 \%$ of the disabled nodes, to make the fitness degree revive to the previous level. In real world, when nodes with emergencies in real cooperation network becomes partially disabled, the emergence of seldom cooperators can soon make up the loss and improve the total fitness degree and cooperative ratio. 


\section{Acknowledgement}

The authors acknowledge the support of the Foundation of Hubei Educational Committee (Grant No. B2013036, 2012G106), and the Youth Science Foundation of Hubei University of Economics (Grant No. XJ201301).

\section{References}

[1] D. J. Watts, S. H. Strogatz, Nature, 393, 440-442 (1998).

[2] A. L. Barabsi, R. Albert, Science, 286, 509-512, (1999).

[3] S. H. Zhang, Ph. D. Thesis, University of Science and Technology of China (Hefei, China), (2006).

[4] J. J. Ramasco, S. N. Dorogovtsev, R. Pastor-Satorras, Phys. Rev. E, 70, 036106, (2004).

[5] L. A. Adamic, B. A. Huberman, Science, 287, 2115, (2000).

[6] M. E. J. Newman, Proc. Natl. Acad. Sci. USA, 98, 404-409, (2001).

[7] X. M. Chen, Q. D. Meng, D. J. Hu, Systems EngineeringTheory \& Practice, 29, 54-62, (2009).

[8] Y. J. Gao, Journal of Xihua University, (Philosophy \& Social Sciences), 23, 29-31, (2004).

[9] M. A. Nowak, S. Bonhoeffer, R. M. May, Proc. Natl. Acod. Sci., USA, 91, 4877-4881 1994.

[10] C. Hauert, G. Szab, Am. J. Phys., 73, 405-414 (2005)

[11] G. Szab, G. Fth, Phys. Rep., 446, 97-216 (2007).

[12] Z. B. Yang, G. Aydin, V. Babich, D. R. Beil, Management Science, 55, 192-209 2009.

[13] A. Nair, R. Narasimhan, T. Y. Choi, Decision Sciences, 40, 783-815 (2009).

[14] A. Szolnoki, G. Szab, Europhys. Lett, 77, 30004 (2007).

[15] R. Axelrod, W. D. Hamilton, Science, 211, 1390-1396 (1981).

[16] F. C. Santos, J. M. Pacheco, J. Evol Biol, 19, 26-733 (2006).

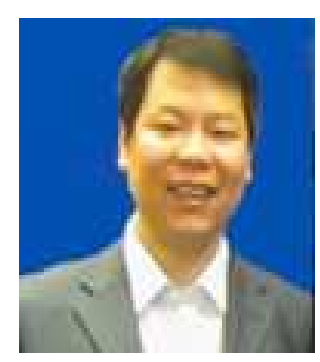

Yaofeng Zhang is a $\mathrm{PhD}$ candidate of the Institute of Systems Engineering at Huazhong University of Science and Technology, Wuhan, China. He has received the $\mathrm{BS}$ degree (2002) in Hebei Normal University and the MS degree (2005) in Xi'an University of Technology. His current research interest focuses on complex system, evolutionary game theory and emergent computation.

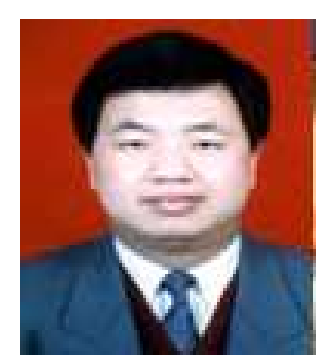

Renbin Xiao is a Professor with the Institute of Systems Engineering, Huazhong University of Science and Technology (HUST). $\mathrm{He}$ is also the Chief Research Scientist in the field of intelligent design and a Professor with the CAD Center, HUST. He received the BS degree in Ship Engineering, the MS degree in Ship Hydrodynamics and the $\mathrm{PhD}$ degree in Systems Engineering from Huazhong University of Science and Technology in 1986, 1989 and 1993, respectively. His research interests include swarm intelligence and emergent computation, management decision theory and decision support system, and creative design of complex products. 